\title{
Vertical Handover in Heterogeneous Networks: a Comparative Experimental and Simulation-based Investigation
}

Giovanni Spigoni, ${ }^{1}$ Stefano Busanelli, ${ }^{2}$ Marco Martalò, ${ }^{1,3}$ Gianluigi Ferrari, ${ }^{1}$ and Nicola Iotti ${ }^{2}$

1: WASN Lab, Dept. Information Engineering, University of Parma, Italy

2: Guglielmo Srl, Pilastro di Langhirano, Parma, Italy

3: E-Campus University, Novedrate (CO), Italy

E-mail: \{giovanni.spigoni,gianluigi.ferrari\}@uniprit

\{stefano.busanelli,nicola.iotti\}@guglielmo.biz

marco.martalo@uniecampus.it

\subsection{Introduction}

The continuously growing traffic generated by Mobile Terminals (MTs) — such as smartphones, tablets, netbooks, and other mobile Internet devices-is nowadays one of the biggest challenges for mobile network operators, especially because this process is not supposed to vanish, at least from a short-time perspective. Therefore, in order to prevent network saturation phenomena, the operators are forced to increase their network capacity more quickly than the customers' demand increase. This goal would be probably achieved by a combination of methods: (i) increasing the available bandwidth in cooperation with public communications agency, e.g., by exploiting the spectrum holes; (ii) increasing the cell spectral efficiency through technology upgrades, e.g., by switching from $3 \mathrm{G}$ to the upcoming Long Term Evolution (LTE) [1] or WiMAX [2] technologies; (iii) reducing the

Heterogeneous Cellular Networks Rose Qingyang Hu and Yi Qian

(c) XXXX John Wiley \& Sons, Ltd 
number of users per macrocell by either reducing the cell size or offloading data traffic through WiFi access points or femtocells [3, 4]. An example of the latter solution, which is of interest in this chapter, can be found in 3G networks at customers' homes, where the deployment of the so-called Home-Evolved Node-B (HeNB) allows to overlap reduced size cells (femtocells) on top of the macrocell of the corresponding Node-B base station [5]. The femtocell configuration is an example of Heterogeneous Network (HetNet), since the involved devices (HeNB and Node-B) have different capabilities (e.g., the coverage range), even if they share the same technology [5]. This configuration is viable if both HeNB and Node-B belong to the same network operator and this could be a serious limitation for its commercial deployment, since the customer has to agree to keep a HeNB in his/her apartment, feeding it with its self-funded Internet connection.

Considering the diffusion of WiFi access points (e.g., IEEE 802.11a/b/g/n [6]), from the customer viewpoint a more attractive solution would consist in jointly using his/her $3 \mathrm{G}$ cellular and WiFi connections. In this case, the devices are different not only in terms of capabilities, as in the femtocell case, but also in terms of technology. Moreover, the UMTS and WiFi networks may belong to two different non-related operators. It is interesting to observe that unlike in a femtocell configuration, the WiFi connection can be used both to replace the $3 \mathrm{G}$ connection (the so-called WiFi offloading [7]) and to increase the bandwidth of the $3 \mathrm{G}$ connection [8]. From the point of view of a mobile network operator, the hybrid UMTS-WiFi solution is more appealing, since its helps reducing the traffic load on the $3 \mathrm{G}$ network. On the other hand, the user has a real advantage only if the WiFi connection can offer an economical saving or a throughput benefit, with respect to the $3 \mathrm{G}$ connection. For this reason, the choice cannot be taken by the operator alone, but the customer has to be, at some extent, involved in the decision process. This is particularly true when the UMTS and WiFi networks belong to two different operators without a specific commercial agreement.

In a classic cellular network, the switch between two different network base stations is governed by a relatively simple Horizontal HandOver (HHO) mechanism [9] and, therefore, it is seamless from the user perspective. In a HetNet, with a single involved technology (e.g., UMTS network with femtocell) $\mathrm{HHO}$ is still possible but it is more complex, especially in the transition from a macrocell to a femtocell. In the case of a hybrid HetNet with two involved technologies, as considered in the current chapter, the complexity is even higher, since it is necessary to use the so-called Vertical HandOver (VHO) mechanisms. The VHO will therefore play a key role in future hybrid HetNets. For this reason, in this chapter we discuss on the potential and the limitations of $\mathrm{VHO}$ in HetNets, on the basis of both experimental and simulation results obtained with two novel low-complexity VHO algorithms. In particular, the experiments are conducted through a small testbed composed by a single MT and a few base stations, whereas the simulation analysis is carried out to investigate large-scale scenarios involving several MTs and base stations.

This chapter is structured as follows. In Section 1.2, we provide the reader with some preliminaries on VHO. In Section 1.3, we present experimental results, with two recently proposed low-complexity VHO algorithms, in a realistic mixed outdoor/indoor scenario. In Section 1.4, an OPNET-based simulator for performance analysis of VHO algorithm is presented: this allows to investigate more complex scenarios with a greater number of MTs. On the basis of the experimental and simulation results, in Section 1.5 we reflect on the practical role of VHO in HetNets. Finally, concluding remarks are given in Section 1.6. 


\subsection{Preliminaries on VHO}

A VHO procedure is composed by three main phases: initiation, decision, and execution [10]. During the initiation phase, the MT (or the network controller) triggers the handover procedure, according to the specific networks' conditions. In the second phase, the VHO algorithm chooses the new access point according to a pre-determined set of metrics, such as the Received Signal Strength Indicator (RSSI), the network connection time, the available bandwidth, the power consumption, the monetary cost, the security level, and, obviously, the user preferences [11]. During the final execution phase, all signaling operations for communication re-establishment and data transfer are carried out. The most relevant international standardization effort regarding VHO and continuous communications, the IEEE 802.21 standard, only refers to the first two phases (initiation and decision) that are relatively technology-independent, but it deliberately ignores the execution phase [12]. Similarly, the Access Network Discovery and Selection Function (ANDSF), defined by the 3GPP [1] consortium, assist user equipment (UE) to discover non-3GPP access networks [13]. The execution phase is the most delicate task of the handover, since it directly impacts with the behavior of the applications running on the MTs. As today, most of the VHO approaches, for example that considered in [14], leverages on some flavors of Mobile IP [15], a level-3 solution that is based on the idea of maintaining the same IP address in every network visited by the MT. For example, the 3GPP consortium leverages on three mobile IP-based protocols: Dual-Stack Mobile IPv6 (DS-MIPv6) [16], Proxy Mobile IPv6 with dual-stack extensions [17], Proxy Mobile IPv6 and Mobile IPv4 (PMIPv6) [18]. However, there is still no universal and definitive solution, and there several works based on different approaches, such as UPMT [19], based on "IP in UDP" tunneling that provides perapplication flow management or based on the application-level Session Initiation Protocol (SIP) [20], mostly because it can better support Voice over IP (VoIP) applications [21].

There are several possible classifications of the VHO algorithms. In particular, they can be distinguished between no-coupling and coupling. The first group of $\mathrm{VHO}$ algorithms refers to scenarios without any form of cooperation between the involved players (users and network operators) [10]. This situation offers the highest degree of freedom to the user, at the price of an increased complexity of the whole handover procedure and of a degraded performance. Clearly, with a higher level of coupling (namely, loose or tight [22]) a better performance can be achieved.

In a no-coupling scenario (the one of interest here and accurately described in Section 1.3), handover times are typically long. Therefore, in order to avoid any lack of connectivity during the handover execution phase, it is necessary to adopt a make-before-break approach. In other words, the old connection is torn down only after the new connection has been established, thus yielding to a period of coexistence of the two connections, during which the MT becomes a temporary multi-homed host. The management of a multi-homed host during the execution phase is an open problem, without a universal solution, and, currently, every Operating System (OS) has its own solution for this problem [23]. 


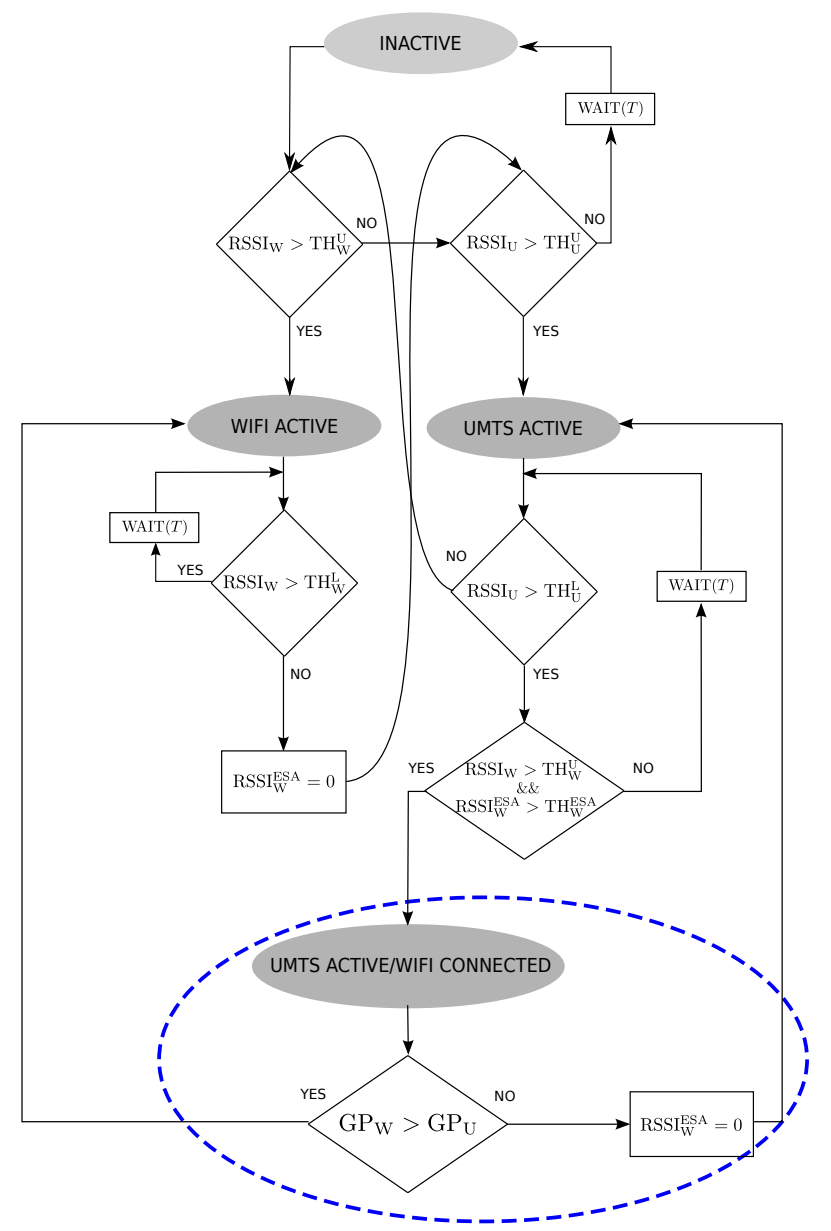

Figure 1.1 Dataflow of the hybrid RSSI/goodput-based VHO algorithm.

\subsection{Experimental Investigation}

\subsubsection{VHO Decision Algorithms}

In [24], two novel low-complexity VHO decision algorithms are proposed. The first decision algorithm is RSSI-based, while the second is a hybrid RSSI/goodput-based decision algorithm. The dataflow of the latter is shown in Figure 1.1, where the portion inside the dotted circle is the only difference with respect to the dataflow of the RSSI-based VHO algorithm. This difference is due to the fact that, while the RSSI-based VHO decision algorithm is based on the implicit assumption that, whenever available, an IEEE 802.11 network always guarantees a better service than a $3 \mathrm{G}$ network, the hybrid RSSI/goodputbased VHO algorithm weighs both RSSI and goodput before making a decision. This extension is motivated to avoid switching from the UMTS network to the WiFi network when the latter offers a smaller effective bandwidth. 
In the RSSI-based VHO algorithm, the instantaneous RSSI value of interface $\mathrm{x}$, denoted as $\mathrm{RSSI}_{\mathrm{x}}$, is compared with two thresholds, denoted as $\mathrm{TH}_{\mathrm{x}}^{\mathrm{U}}$ and $\mathrm{TH}_{\mathrm{x}}^{\mathrm{L}}$. The lower threshold $\mathrm{TH}_{\mathrm{x}}^{\mathrm{L}}$ is used to determine when the RSSI is not sufficient to guarantee a stable connection: therefore, it is slightly higher than the corresponding interface sensitivity. Clearly, when $\mathrm{RSSI}_{\mathrm{x}}<\mathrm{TH}_{\mathrm{x}}^{\mathrm{L}}$ the connection on the interface $\mathrm{x}$ is torn down. On the other hand, the upper threshold $\mathrm{TH}_{\mathrm{x}}^{\mathrm{U}}$ is used to determine if the measured RSSI is sufficient to establish a stable connection. To this end, we assume that $\mathrm{TH}_{\mathrm{x}}^{\mathrm{U}}>\mathrm{TH}_{\mathrm{x}}^{\mathrm{L}}$. The use of two thresholds (per network interface) is the first countermeasure against the ping-pong effect, i.e., the continuous switch between the two networks when the MT moves on the border of the WiFi network coverage region. This is considered as one of the crucial problems in the design of VHO algorithms.

According to Figure 1.1, when in the INACTIVE state, the MT measures, with a period $T$ (dimension: [s]), the RSSI level at each network interface. As soon as the first (of the two) RSSI level overcomes its upper threshold, the corresponding interface notifies the event to the VHO manager, triggering the execution of the Authentication, Authorization, and Accounting (AAA) procedure to join the selected network. We observe that if both networks are available, the priority is always given to the WiFi network. If the AAA procedure in the selected network $x$ succeeds, the state of the MT switches from INACTIVE to "x ACTIVE." Due to the asymmetric nature of the algorithm, the WiFi ACTIVE and the UMTS ACTIVE states have to be treated separately. More details can be found in [24].

In the hybrid RSSI/goodput algorithm, there is an additional state, the WiFi CONNECTED/UMTS ACTIVE state (highlighted at the bottom), where the MT is authorized in both networks. The presence of this state is expedient to estimate the bandwidths of both networks. The bandwidth is estimated by measuring the time necessary to download a 400 kilobyte size file from a remote host (for the ease of simplicity, the file is hosted by a Google server). Another bandwidth estimation technique, well suited to the LTE technology, is based on a more complex algorithm called WBest [25]. The advantage of this algorithm relies on the smaller amount of data downloaded from the server and, thus, on the lower cost of the bandwidth test. This algorithm, in fact, uses a train of packets, sent by the server, to calculate the average delay between the packets introduced by the multi-hop network path. On the basis of this delay and of the size of the packets, it is possible to estimate with sufficient reliability the available bandwidth of a network path with a significantly smaller amount of exchanged bytes with respect to the file download approach-approximately $100 \mathrm{kB}$ against $400 \mathrm{kB}$.

Due to the asymmetric nature of the algorithm, the MT can move towards this new state only from the UMTS ACTIVE state. In particular, during this transition the MT performs the AAA in the IEEE 802.11 network. Then, the MT remains in the WiFi CONNECTED/UMTS ACTIVE state for all the time needed for estimating the goodput of both networks. As soon as the new measurements, denoted respectively as $\mathrm{GP}_{\mathrm{W}}$ and $\mathrm{GP}_{\mathrm{U}}$, are available, the VHO algorithm decides to switch to the WiFi ACTIVE or to come back to the UMTS CONNECTED state. In the latter, the MT disconnects from the WiFi network and resets its RSSI, in order to reduce the waste of resources. From a practical point of view, when $\mathrm{RSSI}_{\mathrm{W}}^{\mathrm{ESA}}>\mathrm{TH}_{\mathrm{W}}^{\mathrm{ESA}}$ the goodput is periodically estimated at a variable, but low, rate, given by the inverse of the sum of the time necessary to complete the AAA procedure and the time necessary to rise again the value of $\operatorname{RSSI}_{\mathrm{W}}^{\mathrm{ESA}}$.

Finally, due to the long time needed by the WiFi AAA procedure, during the UMTS 
ACTIVE $\rightarrow$ WiFi CONNECTED/UMTS ACTIVE transition there are some hidden transitional states, not shown here for the ease of simplicity. In particular, when the AAA procedure fails, the transition to the WiFi CONNECTED/UMTS ACTIVE state cannot be carried out and it is necessary to come back to the UMTS ACTIVE state.

\subsubsection{Experimental Setup and Results}

In [24], the performance of the VHO algorithms described in Subsection 1.3.1 is evaluated in a realistic environment, leveraging on commercially available connectivity service providers and using standard mobile terminal devices. In particular, the experimental test is performed using a notebook running the Windows 7 OS, equipped with a Broadcom IEEE 802.11g compliant network interface and integrated by a UMTS USB Huawei dongle, using the UMTS standard.

The IEEE 802.11 connectivity was offered by a hot-spot owned by one of the biggest Italian Wireless Internet Service Providers (WISPs), namely Guglielmo S.r.l. [26]. The hotspot is given by a Browan IEEE 802.11 access point integrated with a captive portal, while the Authentication Server (AS) is remotely located, as in the standard WISP roaming (WISPr) configuration [27]. The proprietary AAA procedure foresees two additional message exchanges with respect to the WISPr directives [27], thus increasing the time needed to complete the AAA procedure [24]. The UMTS connectivity was instead offered by the Public Land Mobile Network (PLMN) of Telecom Italia, one of the most important Italian mobile operator. The sequence of messages needed to complete the AAA procedure is the same used in a typical $3 \mathrm{G}$ network. We observe that using either WiFi or $3 \mathrm{G}$ connection we have not direct control on the traffic generated by other users.

The two VHO mechanisms described in Subsection 1.3.1 are implemented on top of a so-called Smart Client (SC) software. ${ }^{1}$ According to the WISPR directives, a SC is an application studied for enhancing the user experience, making the AAA procedure automatic. Basically, the goal of the SC is that of constantly monitoring the status of the available connections and executing the VHO. Additionally, once the MT initiates a VHO, the SC automatically has to take care of the suitable AAA procedure. The SC controls both the network interfaces, working with every IEEE 802.11 device able to provide a real time RSSI information, and with every $3 \mathrm{G}$ device (e.g., modem 3G, dongle USB) that supports the Microsoft Remote Access Service (RAS) API [28]. Due to the make-before-break approach, the SC has also to manage the routing functionalities of the OS, in order to make noncritical the multi-homed situation that appears after the authentication on the second network interface [29, 23].

In Figure 1.2, the experimentally estimated (upon time discretization in $0.312 \mathrm{~s}$ bins) Probability Mass Function (PMF) of the handover time is shown. From the results in Figure 1.2, it emerges that the handover times towards WiFi and UMTS networks have very different behaviors. In particular, the PMF of the handover time towards the UMTS network concentrates around its average value $4.13 \mathrm{~s}$ - the standard deviation is $1.76 \mathrm{~s}$. Note that the few values above $10 \mathrm{~s}$ can be considered as outliers. On the other hand, the handover time towards the WiFi network is more "unpredictable" than that towards the UMTS network, as the PMF of the handover time is characterized by a higher average value $(5.43 \mathrm{~s})$ and a much

\footnotetext{
${ }^{1}$ This version of the SC also supports the Microsoft Vista OS. A version for Android-based platforms has also been implemented.
} 

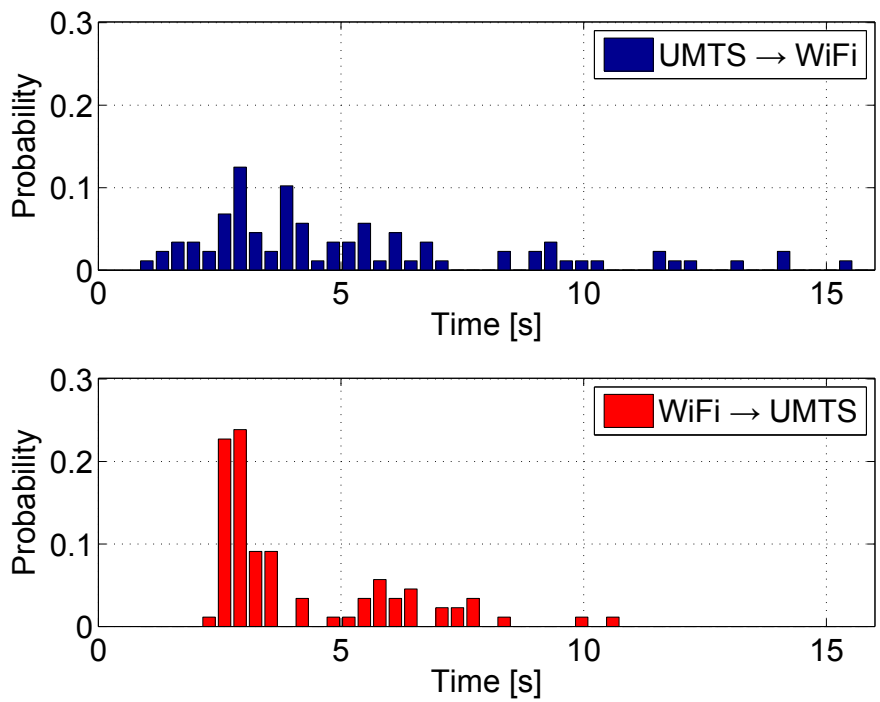

Figure 1.2 PMFs of the handover time of the VHO algorithm presented in Subsection 1.3.1: from UMTS to WiFi (upper) and from WiFi to UMTS (lower).

higher standard deviation $(3.30 \mathrm{~s})$. At the same time, one should observe that the minimum value is very small $(1.22 \mathrm{~s})$.

From the results in the upper subfigure of Figure 1.2, it can be observed that the handover time from the UMTS network to the WiFi network spreads between $1 \mathrm{~s}$ and $10 \mathrm{~s}$. This relatively high variability has several motivations. First of all, in order to save energy, the MT is supposed to logout from a given network once the VHO manager has selected the other network. Sometimes (more often in the WiFi network), the logout fails and the remote authentication server keeps the authentication state for a certain timeout (roughly $60 \mathrm{~s}$ ), before automatically logging the user out. In these cases, frequent UMTS $\rightarrow$ WiFi transitions (in a region at the border of both UMTS and WiFi networks) can experience a short handover time since the MT is de-facto already authenticated to the network. Moreover, while the authentication procedure at MAC layer has, in practice, no impact, the release of an IP address by a DHCP (WiFi network) introduces significant randomness. Finally, when the MT is close to some furniture, the RSSI experiences large oscillations that can delay the AAA procedure. Conversely, the RSSI of the UMTS network is more stable and the probability of experiencing such large variations is very small.

From the results shown in the lower subfigure of Figure 1.2, it can be observed that the handover time from the WiFi network to the UMTS network is generally shorter and more predictable (i.e., its PMF is more concentrated) than that in the opposite direction.

However, due to the no-coupling and the lack of any optimization, the handover time may be long also in the transition towards the UMTS network. This result has somehow to be expected, since the proposed VHO algorithm is designed to be used for slowly mobile MT, e.g., people moving from a place to another.

In order to measure the goodput, we focus on a single walking path, chosen among the 


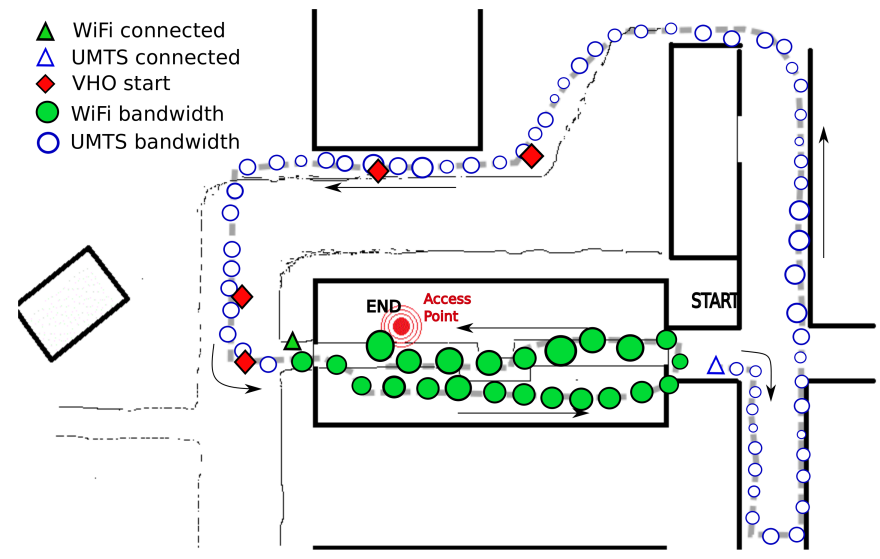

Figure 1.3 Throughput improvement using the hybrid RSSI/goodput-based algorithm.

experimental data set. The selected path is shown in Figure 1.3 (a), along with the layout of the environment where the tests were performed. Our experiments were conducted in a building within the Department of Information Engineering of the University of Parma. The nearest UMTS base station is placed roughly $1 \mathrm{~km}$ far away from the building, and it offers a 7.2 Mbit/s downlink (384 kbit/s uplink) bandwidth, being compliant with the UMTS specifications. We have placed the hotspot in the WASN Lab, at $1 \mathrm{~m}$ above the ground. The hotspot is fed by an optical fiber network with $100 \mathrm{Mbit} / \mathrm{s}$ of symmetric bandwidth, but the hotspot imposes a symmetric limit on the available bandwidth equal to 7.2 Mbit/s, similar to that provided to typical customers. Despite the identical nominal downlink UMTS bandwidth (7.2 Mbit/s), the WiFi network has often outperformed, in our tests, the UMTS network.

The test were performed by walking through the building, keeping the notebook in hand and measuring (i) the time needed to perform the handover and (ii) the goodput variations. The tests were always performed during working hours, in order to obtain results associated with realistic daylife situations.

The bold solid lines represent reinforced concrete walls, that cause strong signal attenuation. In correspondence to a glass window or a door (where the bold solid lines are interrupted), the signal attenuation is clearly much weaker. The path followed by the user is represented by a dashed line and is delimited by the words "START" and "END." The circles drawn along the path represent the measured available goodput: in particular, the diameter of the circle is proportional to the available goodput. In correspondence to filled circles, data was sent via the IEEE 802.11 interface, while in correspondence to empty circles the UMTS interface was used.

A (filled) diamond denotes the beginning of a VHO procedure, while a triangle indicates when the procedure has been successfully completed. The filled triangles indicate that the VHO procedure has established a WiFi connection, while empty triangles denote the establishment of a UMTS connection. We stress the fact that between diamonds and triangles the MT is still connected with the old network, in order to avoid partial loss of connectivity before finalizing the VHO. Finally, the distance between the circles is directly proportional to the duration of the bandwidth test and, hence, inversely proportional to the available bandwidth. In Figure 1.4, the RSSI and goodput relative to the hybrid RSSI/goodput VHO 

Investigation $\quad 9$
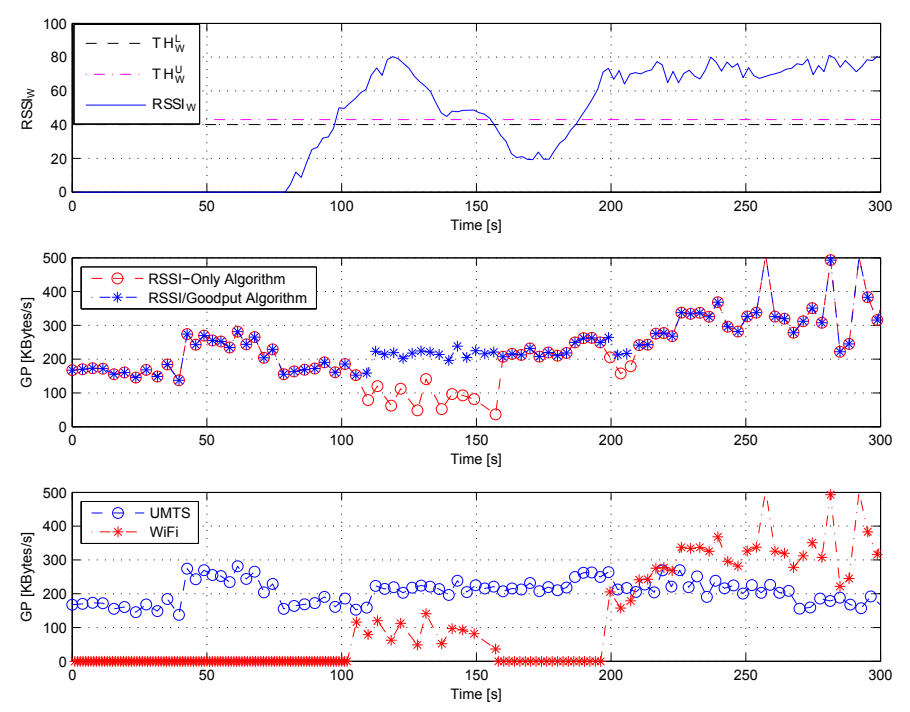

Figure 1.4 RSSI and goodput experienced by the MT following a sample path using the hybrid RSSI/goodput VHO algorithm.

algorithm, along a sample path of about $200 \mathrm{~m}$ (partly indoor and partly outdoor), are shown as functions of time. For the sake of clarity, a direct comparison with the RSSIbased VHO algorithm is also considered. In the upmost subfigure, RSSI $\mathrm{W}_{\mathrm{W}}$ is shown together with the corresponding upper and lower thresholds; in the lowest subfigure, the estimated goodputs $\mathrm{GP}_{\mathrm{W}}$ and $\mathrm{GP}_{\mathrm{U}}$ are directly compared; finally, in the middle subfigure the overall goodput guaranteed by the RSSI-based VHO algorithm (denoted as GP) and the hybrid RSSI/goodput-based VHO algorithm (denoted ad $\mathrm{GP}^{H}$ ) are directly compared. According to the results in Figure 1.4, in the initial phase the MT is disconnected from the WiFi network, because of the too low received power. In the upmost subfigure, it can be observed that at a given point (a particular position along the path), $\mathrm{RSSI}_{\mathrm{W}}$ starts to quickly increase and soon overcomes the threshold $\mathrm{TH}_{\mathrm{W}}^{\mathrm{U}}$. At this moment, the RSSI-based algorithm begins the VHO towards the WiFi network, ignoring the fact that the effective goodput available in the WiFi network is lower (as can be seen in the lowest subfigure). On the other hand, in the case of the hybrid VHO algorithm, the MT starts the bandwidth estimation process, after which it decides to keep the UMTS connection because it becomes aware of its higher available goodput. By observing the middle subfigure, the hybrid RSSI/goodput-based VHO algorithm guarantees a better (over time) goodput performance than that of the RSSI-based algorithm, the only "penalty" being slightly higher handover time and complexity. These prices to be paid are due to the presence of a double connectivity situation, which requires to properly configure the OS routing table, in order to perform the bandwidth test on both networks, without penalizing the user. 


\subsection{Simulation-based Investigation}

In this section, we present a simulation-based investigation of the proposed VHO algorithms in HetNet scenarios by relying on the Opnet simulator [30]. We first examine a scenario where a single node is moving across the network, in order to reproduce and validate the experimental results presented in Subsection 1.3.2. However, as it will be described in more detail in the next subsection, the current version of the simulated VHO algorithm between WiFi and UMTS networks is only based on the received power of the WiFi interface. The UMTS signal, in fact, is assumed to be always present, since in realistic urban scenarios one can assume that a $3 \mathrm{G}$ connection is available at every time. Then, we will extend the analyzed scenario, considering a large number of nodes randomly moving in the HetNet and carrying out, possibly, VHO procedures. Note also that the realistic AAA procedures for $3 \mathrm{G}$ and WiFi networks are not exactly replicated in the simulator, but their effects are reproduced by adding, during the $\mathrm{VHO}$ process, a random delay drawn from the PMF shown in Figure 1.2.

The performance of the hybrid RSSI/goodput-based VHO algorithm has also been investigated in the presence of an LTE cellular network. Considering an LTE system, at the place of a $3 G$ system, can be interesting as LTE systems are being deployed in many countries and, therefore, the performance analysis with this technology is timely. In order to analyze the VHO between WiFi and LTE networks, we created a scenario where 40 mobile nodes move randomly across the coverage area of an LTE base station, where there is also a WiFi access point. All the mobile nodes implement the hybrid VHO algorithm.

\subsubsection{The OPNET Simulator}

OPNET is a modular discrete-event simulator providing support for several technologies, among many others, WiFi, UMTS, and LTE networks. The WiFi implementation adheres to the IEEE 802.11g standard, whereas the UMTS implementation, supposed to be compliant with the 3GPP Release 5, does not support HSDPA and it offers a maximum downlink throughput equal to $384 \mathrm{kbps}$ (the HSDPA maximal nominal throughput is instead equal to $14.4 \mathrm{Mbit}$ ). The LTE implementation is compliant with the 3GPP Releases 8 and 9: for instance, it is possible to select the number of antennas of the devices in the MultipleInput Multiple-Output (MIMO) setting and pre-set the spectrum bandwidth. Note that the LTE system provides a high data-rate downlink connection which can be compared with that of a HSPA+-equipped 3G system. ${ }^{2}$ We point out that a comparison between WiFi and UMTS (without HSPA) available bandwidths wouldn't make sense, since the WiFi data-rate is (almost always) much higher than the UMTS data-rate.

In all the networks of interest, the MTs implement the entire protocol stack, also including the application layer on which desired applications can be run. In particular, we consider three downlink scenarios. In the first two scenarios, there is a File Transfer Protocol (FTP) application running and each node downloads, every second from an FTP server, a file of a given size, which depends on the scenario of interest. In the second scenario,

\footnotetext{
${ }^{2}$ Downlink connection data-rates for HSPA+ and LTE are not the same, although both technologies provide several tens of Mbps depending on the physical configuration (multiple-antenna techniques, spectrum bandwidth, multi-cell technique, terminal category, etc.)
} 


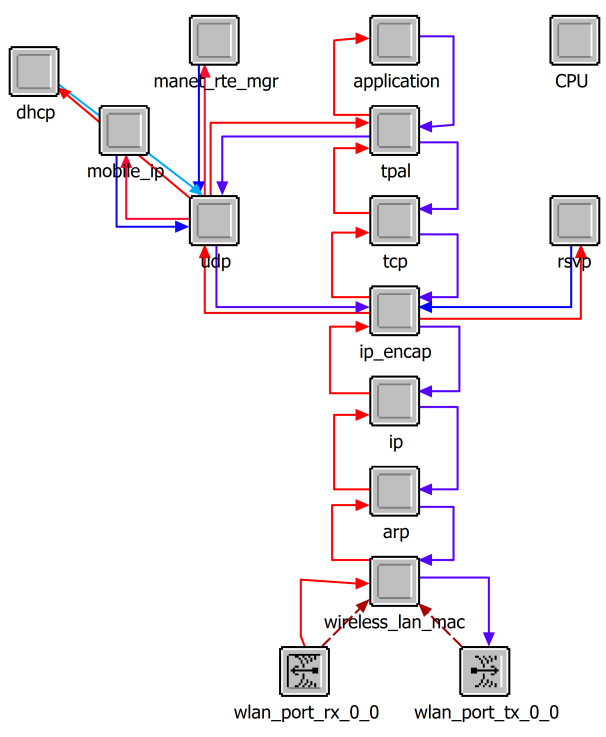

WIFI PROTOCOL STACK

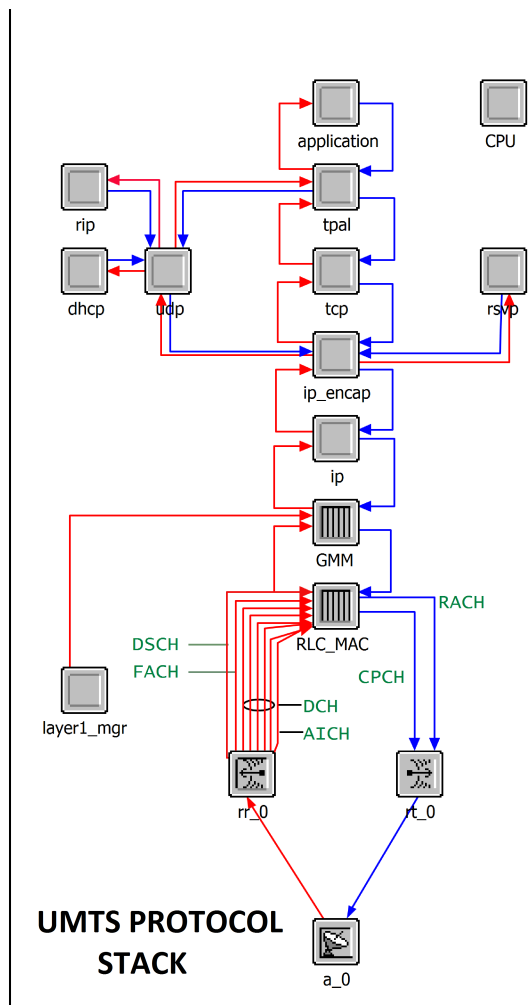

Figure 1.5 Protocol stack of a WiFi node and a UMTS node in the OPNET simulator.

the MTs launch their applications one at a time every $10 \mathrm{~s}$. The first MT waits $100 \mathrm{~s}$ before running its application, in order to let every MT to associate to the UMTS network. In the last scenario, instead, each node executes, every $60 \mathrm{~s}$ on average, a Hypertext Transfer Protocol (HTTP) application downloading a web page of a given size. The LTE MIMO configuration is set to $2 \times 1$ (two transmitter antennas and one receiver antenna) for the downlink and to $1 \times 2$ for the uplink. The spectrum bandwidth is set to $20 \mathrm{MHz}$. Wireless communications between MTs and APs (or base stations) follow a signal propagation model where fading with a Ricean distribution is also included. However, in the considered settings, the impairments due to fading are relatively small and, therefore, the signal attenuation is very similar to a free-space model.

The main challenge in simulating a VHO algorithm is the implementation of a node module able to jointly control both a WiFi and a cellular (UMTS or LTE) radio interface. In our simulator, for the couple WiFi/UMTS this goal has been pragmatically achieved by coupling two independents nodes, equipped, respectively, with a WiFi and an UMTS network interface and the corresponding protocol stack, as shown in Figure 1.5. However, since the nodes are forced to move together through the network at a fixed distance of $10 \mathrm{~cm}$ from each other, they appear, from a network perspective, as a single node. 


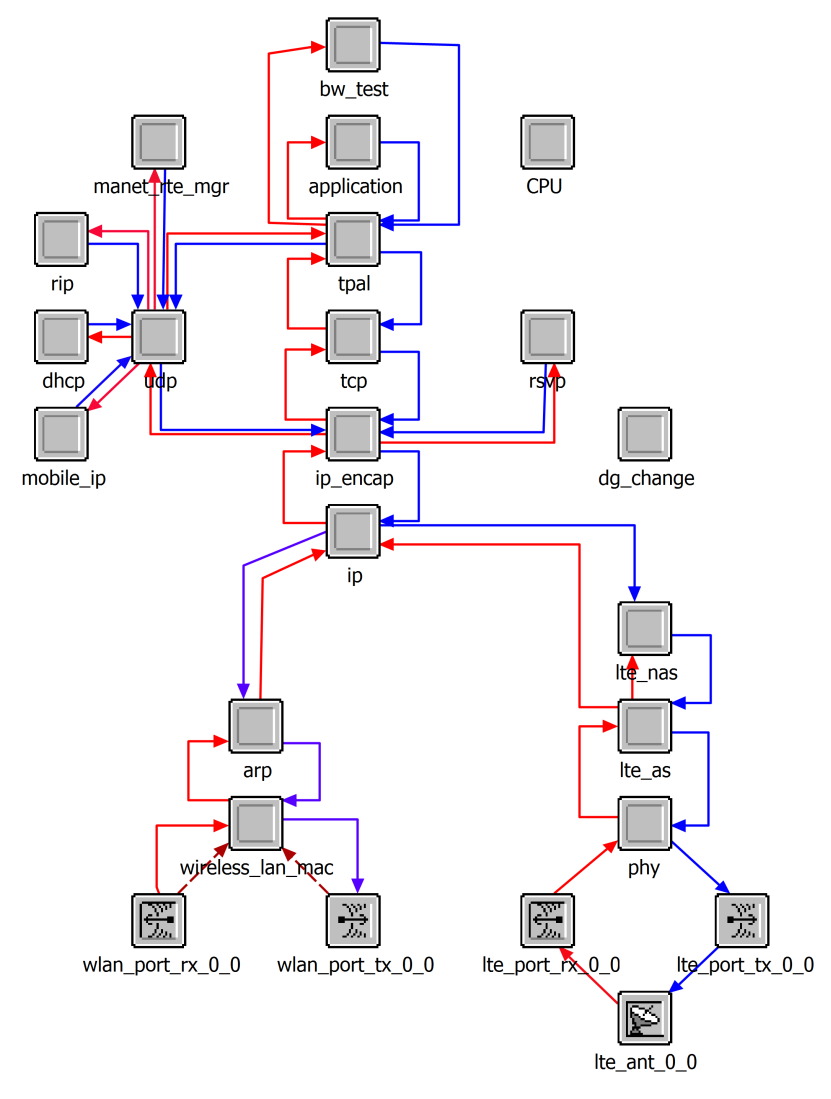

Figure 1.6 Protocol stack of the new node with WiFi and LTE interfaces developed with OPNET modeler. The module $b w$ test is responsible for the computation of the available bandwidth estimation, while the $d g$ _change module manipulates the IP routing table in order to change the default gateway and to redirect specific traffic over a given network.

The VHO algorithm is implemented at the MAC layer of the WiFi node, i.e., in the module denoted as wireless_lan_mac in Figure 1.5. As already anticipated at the beginning of this section, the power level of the UMTS signal is assumed to be always greater than $T H_{U}^{U}$. Moreover, the power of a beacon frame sent by the WiFi access point is computed and filtered as described in Subsection 1.3.1. If the conditions triggering the VHO procedure represented in Figure 1.1 are verified, the MAC layer module sends an interrupt to the application layer of both WiFi and UMTS nodes. In our simulator, the threshold is set to $-83 \mathrm{dBm}$. If the VHO is towards the WiFi network (i.e., if $\mathrm{RSSI}_{\mathrm{W}}>\mathrm{TH}_{\mathrm{W}}^{\mathrm{U}}$ and $\mathrm{RSSI}_{\mathrm{W}}^{\mathrm{ESA}}>\mathrm{TH}_{\mathrm{W}}^{\mathrm{ESA}}$ ), the FTP application on the WiFi node starts receiving data. On the other hand, the application on the UMTS node stops the data generation, in order to simulate a hard disconnection at this interface. The opposite operations are performed if the VHO is towards the UMTS network.

In the case of WiFi/LTE VHO, we have developed a new Opnet node having both the WiFi and LTE interfaces as shown in Figure 1.6. In this node, the $d g_{\text {_change module is }}$ 
responsible for the commutation of the traffic between WiFi and LTE interfaces. The wireless_lan_mac module computes the WiFi power level as explained in the previous paragraph, while the $b w$ test module executes the available bandwidth (in both WiFi and LTE networks) estimation. Both the bw_test and the wireless_lan_mac modules communicate their metrics to the $d g \_$change module that, if necessary, triggers a VHO. In this case, the WBest-based bandwidth estimation algorithm is applied.

\subsubsection{Performance Results}

In this subsection, we analyze the performance predicted by the OPNET simulator for three possible scenarios of interest. In the first case, we consider the same scenario used to experimentally validate the VHO algorithm and shown in Figure 1.3: a single node moves, for several times, from the WiFi coverage area to the UMTS cell and vice-versa. This scenario is expedient to verify, trend-wise, the experimental results presented in Section 1.3 and, therefore, to obtain a sanity-check of our simulator. In the second case, 40 nodes randomly move inside the same area of interest. A unique WiFi access point is placed in this area, whereas four UMTS antennas are placed to ensure total cellular coverage over the entire area. This is representative of a realistic scenario where many users may be in the same city area close to an access point (e.g., a crowded square) and may want to connect to the Internet (e.g., through their smartphones). In all simulations, the metrics are measured starting from the end of the initial transient, of duration equal to $100 \mathrm{~s}$, in order to allow the UMTS MTs to perform the network association procedure. The third scenario aims at validating the experimental results obtained with the hybrid VHO algorithm. In this scenario, a WiFi access point and an LTE eNodeB (i.e., an LTE base station) are placed in the same region: the coverage area of the $L T E$ eNodeB has a $1 \mathrm{~km}$ radius, while the WiFi range is about $400 \mathrm{~m}$. In every scenario, the performance metric of interest is the goodput, defined as the total received traffic (dimension: [bytes/s]) at application layer.

\section{Single Node Scenario: UMTS and WiFi networks}

The first scenario is shown in Figure 1.7. In this case, both applications try to periodically (each second) download from the FTP server a file whose dimension is interface-dependent: $35 \mathrm{kB}$ from the UMTS interface and $50 \mathrm{kB}$ from the WiFi interface. This difference in file size has the sole purpose to differentiate in the graph the UMTS traffic from WiFi traffic. The node moves on the deterministic path, highlighted in Figure 1.7 and representative of the realistic one considered in Figure 1.3, at a constant speed $v=2 \mathrm{~m} / \mathrm{s}$. The total length of the path is approximately $1.4 \mathrm{Km}$.

In Figure 1.8 (a), the received power (in $\mathrm{dBm}$ ) of the beacon frames is shown. Beacon frames are sent by the WiFi AP every 0.5 seconds. In this simulated scenario, the curve is smoother than the corresponding curve in Figure 1.4. This is due to the fact that, unlike the experimental scenario where the signal is impaired by the reflections of the buildings, in the simulated scenario only reflections of the ground are considered, therefore the effects on the signal are very similar to that of a free-space scenario. In Figure 1.8 (b), the goodput, due to either WiFi or UMTS connections, of the node moving along the path described in Figure 1.7 is shown as a function of time. As one can see, every time a beacon frame is received from the WiFi access point, as shown in Figure 1.8 (a), the VHO algorithm is triggered and the 
14 Vertical Handover in Heterogeneous Networks: a Comparative Experimental and Simulationbased Investigation

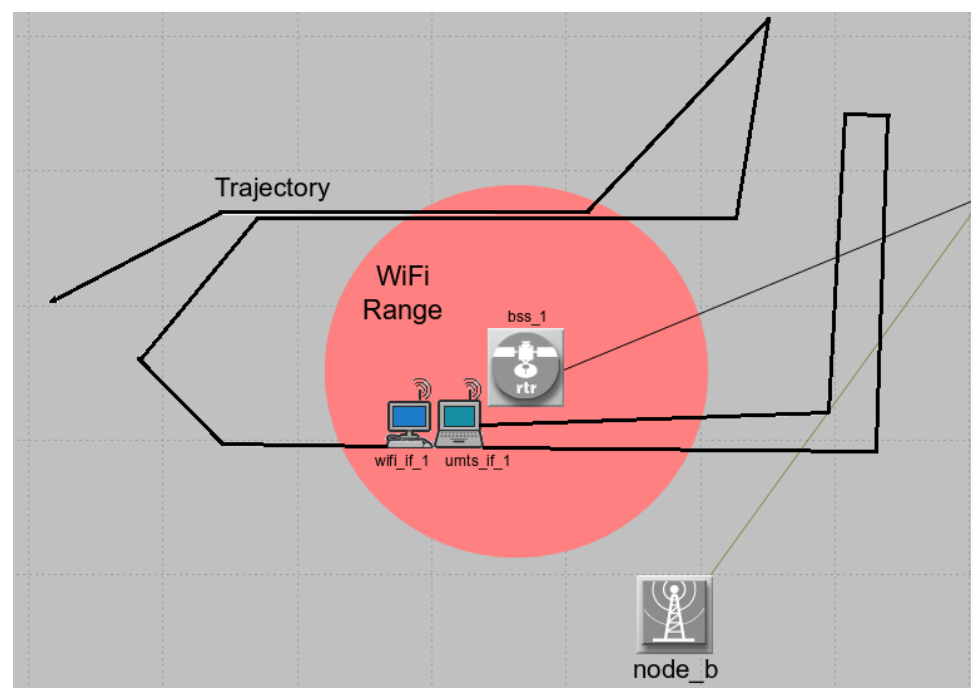

Figure 1.7 First simulation scenario with a single MT following a deterministic path.

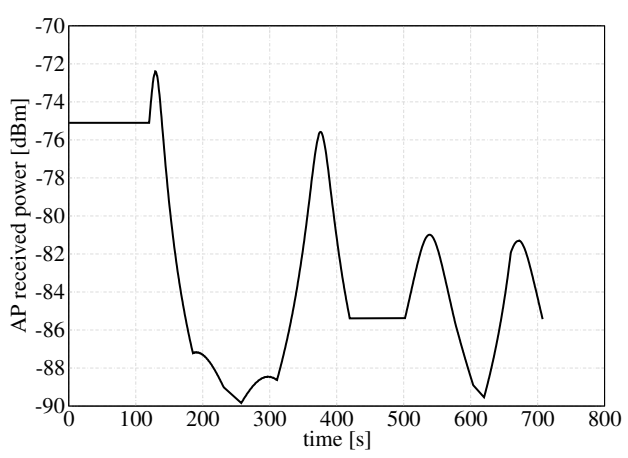

(a)

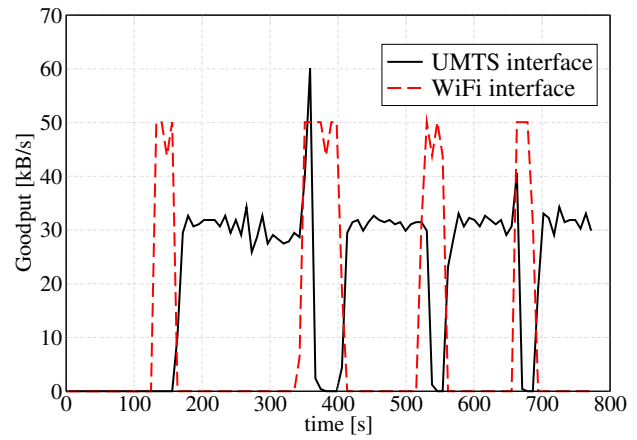

(b)

Figure 1.8 (a) Received power on the WiFi interface and (b) goodput on both the interfaces for the single node scenario.

goodput on the WiFi interface increases, whereas the UMTS interface is triggered down. We recall that the threshold on the received power is set, in our simulator, to $-83 \mathrm{dBm}$. This confirms the experimentally acquired results shown in Section 1.3.

\section{Multiple Node Scenario: UMTS and WiFi networks}

In Figure 1.9, the second simulated scenario of interest is shown. In this case, there are 40 MTs randomly moving in the depicted square area of interest. The MTs move according to the Random Waypoint mobility model [31] with a speed uniformly distributed in the interval 


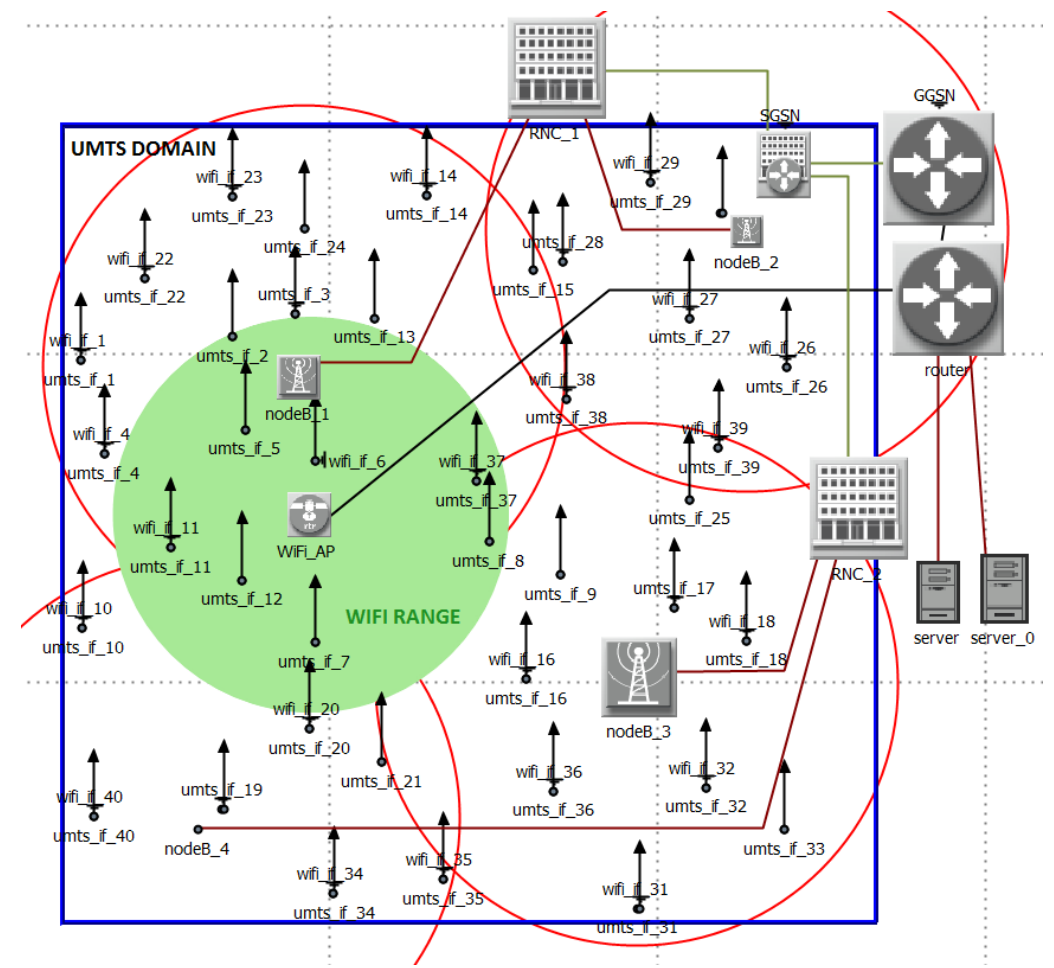

Figure 1.9 Second simulation scenario with multiple MTs moving according to the Random Waypoint mobility model. MTs have both WiFi and UMTS interfaces.

between 0 and $5 \mathrm{~m} / \mathrm{s}$. The WiFi AP is placed inside the coverage UMTS area: when MTs get sufficiently close to the AP, they stop the UMTS connection and start sending and received data through the WiFi interface. The small filled circle represents the WiFi signal range, corresponding to a transmission power equal to $7 \mathrm{dBm}$ (approximately $5 \mathrm{~mW}$ ). Every MT tries to download, every second, a file of $30 \mathrm{kB}$ so that the total goodput received by the 40 MTs should be $1200 \mathrm{kB} / \mathrm{s}$. The number of UMTS base stations is set to 4 to ensure UMTS connectivity for all MTs moving inside the perimeter denoted as "UMTS DOMAIN." The Radio Network Controllers (RNCs) and the core network nodes (SGSN and GGSN) of the UMTS system are also shown, as well as the two FTP servers.

In Figure 1.10, we show (a) the goodput and (b) the corresponding number of connected MTs as functions of time. Sixty independent simulation runs were performed to eliminate statistical fluctuations of the results. In all simulations, the metrics are measured starting from the end of the initial transient, of duration equal to $100 \mathrm{~s}$, in order to allow the UMTS MTs to perform the network association procedure. One can first observe that the goodput is an increasing function of the time. This is due to the fact that, as time passes, the number of MTs in the network increases and, therefore, the total received traffic on each (or both) the interfaces becomes higher. Moreover, from Figure 1.10 (a), one can see that when both the MTs' interfaces are active, the total received goodput approaches $1000 \mathrm{kB} / \mathrm{s}$, which is 
16 Vertical Handover in Heterogeneous Networks: a Comparative Experimental and Simulationbased Investigation

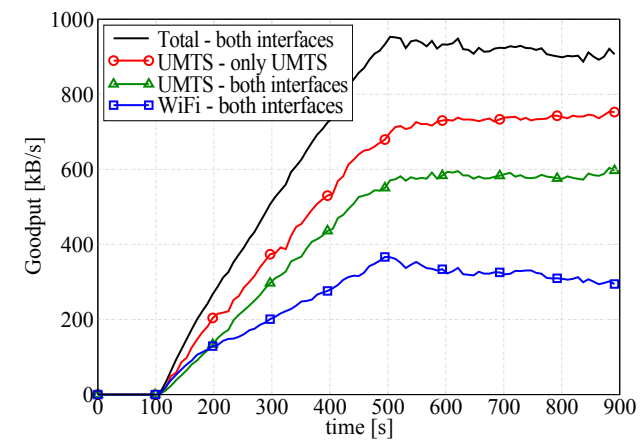

(a)

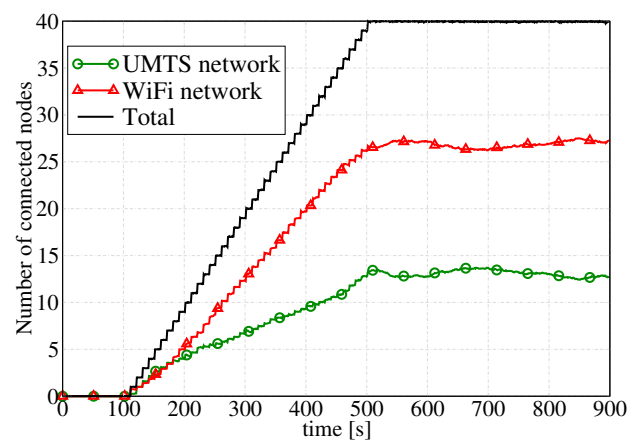

(b)

Figure 1.10 (a) Goodput and (b) number of the transmitting MTs in each network for the multiple node scenario.

close to the maximum possible value of $1200 \mathrm{kB} / \mathrm{s}$. On the other hand, when only the UMTS interface is active, the traffic saturates to approximately $800 \mathrm{kB} / \mathrm{s}$. A similar result holds for the scenario where both interfaces are active. Therefore, the presence of a WiFi connection supplies further connectivity needed to reach the (theoretical) highest possible goodput.

\section{Multiple Node Scenario: LTE and WiFi networks}

In Figure 1.11, the third simulated scenario of interest is shown. In this case as well, there are 40 MTs moving across the area depicted by a blue quadrangle, the filled circle being the coverage area of the WiFi access point. The mobility model and the maximum speed of the nodes are the same as the ones in the second scenario. Both WiFi access point and LTE system (eNodeB and Evolved Packet Core, EPC) are connected, as well as 4 HTTP servers, to the Internet cloud.

In Figure 1.12, the estimated bandwidth is shown, as a function of time, for a single node in the network. For the first part of the simulation, both WiFi and LTE network have no traffic to be delivered and, therefore, the estimated bandwidth corresponds to the maximum capacity offered by the networks. After approximately nine minutes, some traffic is generated on the LTE network and, after a few seconds, the MT detects the strong decrease of available bandwidth over the LTE network. Consequently, the MT executes a VHO procedure towards the WiFi network.

In Figure 1.13, the performance of the hybrid VHO algorithm is compared, in terms of total aggregated goodput (dimension: [bytes/s]), with that of the RSSI-based algorithm. The results are obtained executing 16 15-minute long simulation runseight runs for the RSSI-based algorithm and eight runs for the hybrid algorithm-of the third scenario. The network increases at each simulation run, due to the fact that the average size of the downloaded HTTP pages increases. For every run, we compute the average aggregated goodput of the networks. Note that the total load is increased in order to saturate both the WiFi and the LTE networks. In particular, the WiFi bandwidth saturates before the LTE network is overloaded-this is obtained by setting the WiFi range so that the number of nodes within the WiFi coverage is sufficiently 


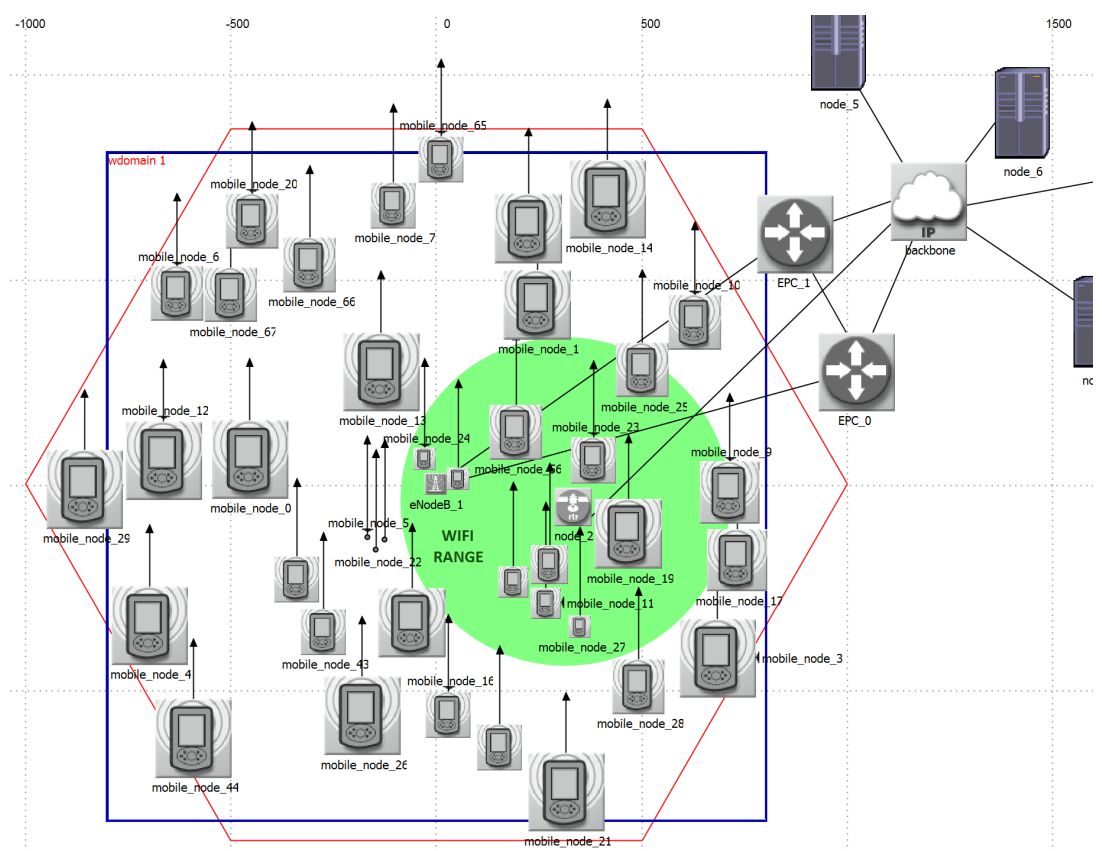

Figure 1.11 Third simulation scenario with multiple MTs moving according to the Random Waypoint mobility model. MTs have both WiFi and LTE interfaces.

large. One can see that the goodput delivered through the WiFi interface does not change considerably between the hybrid and the RSSI-based algorithms. ${ }^{3}$ The most noticeable difference between the two algorithms can be observed through the LTE interface. Obviously, the goodput reached by the LTE network in saturation conditions does not change. However, with the hybrid algorithm the goodput increases faster, due to the fact that, when the WiFi can no longer transport further data and the LTE band is not overloaded, the nodes under WiFi coverage switch their connections towards LTE. This fact could be seen as a load balancing between LTE and WiFi implicitly executed by the VHO hybrid algorithm. In fact, thanks to the bandwidth estimation metric, the mobile nodes are somehow aware of the saturation of the WiFi network and can select the LTE connection.

For the sake of completeness, we point out that the total goodput is never equal to the total load requested by the mobile nodes. This is due to the implementation of the HTTP application: the time interval between two HTTP requests is not keep fixed to $60 \mathrm{~s}$, but it has an exponential distribution with mean value equal to $60 \mathrm{~s}$. Therefore, it may happen that an HTTP request arrives when the previous request (for the same MT) has not been served. At this point, the HTTP client interrupts the download in progress to start the new one. The total offered load does not take into account this reduction on

\footnotetext{
${ }^{3} \mathrm{~A}$ slight decrease can be observed in the case of RSSI-based algorithm due to the increasing number of packets collision at WiFi physical layer.
} 
18 Vertical Handover in Heterogeneous Networks: a Comparative Experimental and Simulationbased Investigation

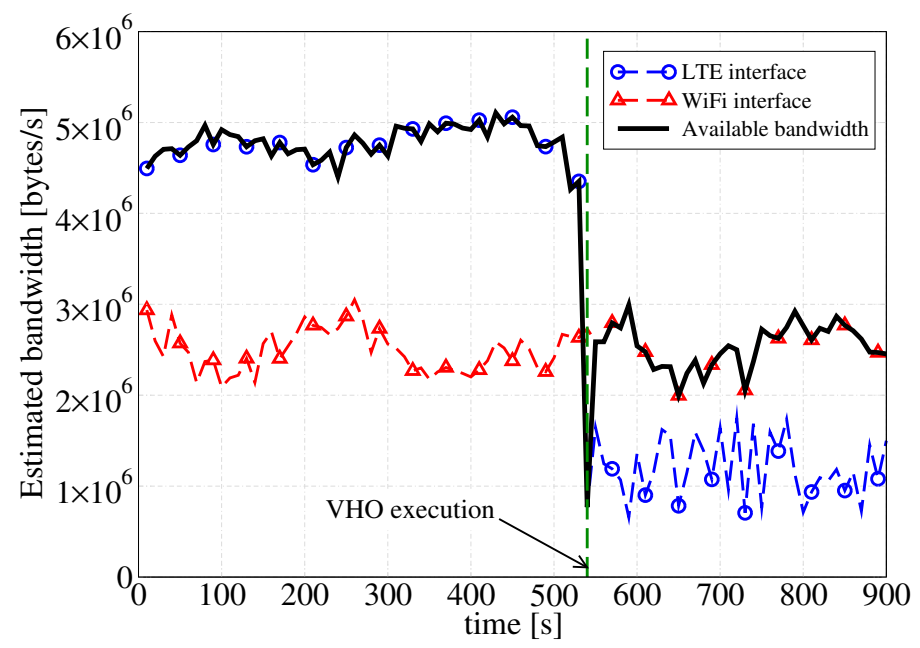

Figure 1.12 Estimated bandwidth, as a function of time, for a single node in the third scenario.

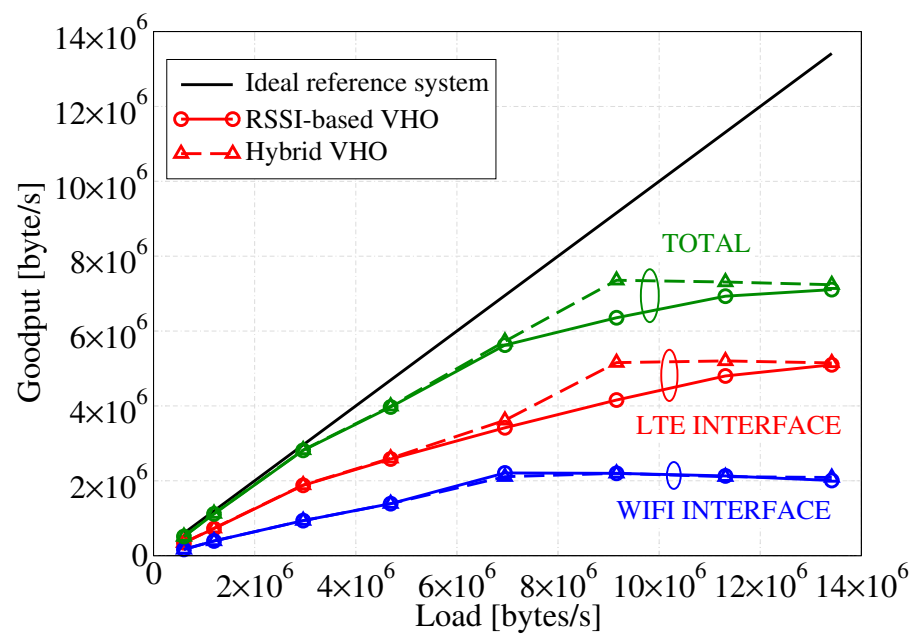

Figure 1.13 Total aggregated goodput of the networks for both the hybrid and the RSSI-based VHO algorithms.

the requested data.

\subsection{Discussion on the VHO in HetNets}

On the basis of the experimental and simulation results presented and discussed in Section 1.3 and Section 1.4, respectively, the following reflections on the role of VHO in HetNets can be carried out. 


\subsubsection{Role of the (Internal) Decision Algorithm}

From the obtained experimental results, it turns out that the VHO decision algorithm is relevant to mitigate the ping-pong effect. However, the RSSI/goodput-based decision algorithms guarantee a very good performance, provided that the received QoS indicator (either the RSSI or a running estimate of the available goodput) is properly filtered, in order to avoid sudden (and temporary) handover decisions.

\subsubsection{Role of the Authentication Procedures}

The authentication procedure seems to be the real bottleneck of the VHO procedure, especially in a no-coupling scenario, where the MT needs to switch between different operators. In this case, the only solution is a radical simplification of this procedure, possibly demanding some security mechanism to lower levels of the protocol stack. On the other hand, in the case of tight coupling, where the two networks involved in the VHO are operated by the same provider, it might be possible to significantly simplify the authentication procedure, thus reducing the VHO time.

\subsubsection{Impact of VHO on HetNet Coverage}

The impact of the VHO on network coverage depends on the networks between which it is carried out. More precisely, in the case of two heterogeneous networks with radically different coverage ranges (e.g., UMTS and $\mathrm{WiFi}$ ), the VHO procedure has basically no impact on the coverage extension, but it is mainly expedient to select, among two simultaneously available networks, the one which guarantees the highest QoS-according to the chosen performance indicator. On the other hand, VHO might play a key role to efficiently extend the coverage when carried out between networks with more similar coverage ranges, e.g., UMTS and WiMAX networks. The same experimental investigation described in this chapter can be applied to different pairs of networks as well, provided that proper connection procedures and VHO algorithms are implemented in the SC.

\subsubsection{Impact of VHO on HetNet Capacity}

The impact of VHO on the capacity of a HetNet is not easy to evaluate. In fact, an efficient VHO mechanism allows each MT to connect to the best currently available network. In particular, if the chosen QoS indicator is the available goodput, the selection, by each node, of the network which guarantees the highest goodput implies, from a single-user perspective, maximization of the capacity. Roughly speaking, Figure 1.4 shows that the choice of the network with the best available goodput allows to double the bandwidth experienced by a single MT. In general, VHO will likely be a key ingredient to perform efficient cellular offloading [8] and will thus have a crucial role in $4 \mathrm{G}$ systems [11].

However, as known in the realm of game theory, the maximization of each user's utility does not necessarily imply the maximization of the entire HetNet utility [32]. In fact, there may be a large number of MTs which would like to connect to the network with the best goodput (e.g., the WiFi network), thus leading to a violation of the minimum QoS on this network and, therefore, to a congestion. This opens several interesting research perspective, 
as it is expected that a centralized control of a HetNet will really allow to exploit its potential. On the other hand, efficient decentralized control strategies will likely play a key role in future systems.

\subsection{Conclusions}

In this chapter, we have reflected on the role of VHO in future HetNets. In particular, on the basis of internetworking experimental results obtained with low-complexity novel VHO algorithms (relying on RSSI and goodput measurements) [24], we have drawn some simple conclusions on the potential and limitations of VHO in HetNets. The main conclusion is that the VHO procedure in loosely-coupled heterogeneous networks experiences a high handover time, mostly due to the latency induced by the AAA procedures. Therefore, the design of effective VHO mechanisms requires to consider a top-down interaction from the high layers of the protocol stack to the bottom layers of the same. Moreover, the simulation-based investigation has shown that the use of VHO has the potential to perform cellular offloading, thus increasing network capacity.

\section{Acknowledgment}

This work is sponsored in part by Guglielmo Srl and in part by the project "Cross-Network Effective Traffic Alerts Dissemination” (X-NETAD, Eureka Label E! 6252 [33]), sponsored by the Ministry of Foreign Affairs (Italy) and the Israeli Industry Center for R\&D (Israel) under the "Israel-Italy Joint Innovation Program for Industrial, Scientific and Technological Cooperation in R\&D."

We would like to thank Ing. G. Guerri (Guglielmo Srl) for his continuous support and help.

\section{References}

[1] 3rd Generation Partnership Project, "3GPP," Website: http://www.3gpp.org.

[2] Insitute of Electrical and Electronics Engineers, "IEEE Std 802.16TM-2009. Part 16: Air Interface for Broadband Wireless Access Systems," 2009.

[3] J. Andrews, H. Claussen, M. Dohler, S. Rangan, and M. Reed, "Femtocells: Past, present, and future," IEEE J. Select. Areas Commun., vol. 30, no. 3, pp. 497-508, April 2012.

[4] V. Chandrasekhar, J. Andrews, and A. Gatherer, "Femtocell networks: a survey," IEEE Commun. Mag., vol. 46, no. 9, pp. $59-67$, September 2008.

[5] A. Ghosh, N. Mangalvedhe, R. Ratasuk, B. Mondal, M. Cudak, E. Visotsky, T. Thomas, J. Andrews, P. Xia H. Jo, H. Dhillon, and T. Novlan, "Heterogeneous cellular networks: From theory to practice," IEEE Commun. Mag., vol. 50, no. 6, pp. 54-64, June 2012.

[6] Insitute of Electrical and Electronics Engineers, "IEEE Std 802.11TM-2007. Part 11: Wireless LAN Medium Access Control (MAC) and Physical Layer (PHY) specifications," 2007.

[7] K. Lee, I. Rhee, J. Lee, Y. Yi, and S. Chong, "Mobile data offloading: how much can WiFi deliver?" SIGCOMM Comput. Commun. Rev., vol. 40, no. 4, pp. 425-426, October 2010.

[8] A. Balasubramanian, R. Mahajan, and A. Venkataramani, "Augmenting mobile 3G using WiFi," in Proc. of the Int. Conf. on Mobile Systems, Applications, and Services (MobiSys), San Francisco, CA, USA, June 2010, pp. 209-222.

[9] J. Manner, M. Kojo, T. Suihko, P. Eardley, and D. Wisely, "IETF RFC 3753, Mobility related terminology," 2004.

[10] G. Lampropoulos, N. Passas, L. Merakos, and A. Kaloxylos, "Handover management architectures in integrated WLAN/cellular networks," IEEE Communications Surveys \& Tutorials, vol. 7, no. 4, pp. 30-44, October 2005 . 
Vertical Handover in Heterogeneous Networks: a Comparative Experimental and Simulation-based Investigation 21

[11] X. Yan, Y. A. Sekercioglu, and S.Narayanan, "A survey of vertical handover decision algorithms in 4G heterogeneous wireless networks," Elsevier Computer Networks, vol. 54, no. 11, pp. 1848-1863, August 2010.

[12] Insitute of Electrical and Electronics Engineers, "IEEE Std 802.12TM-2008. Part 21: Media Independent Handover Services," 2008.

[13] J. Sachs and M. Olsson, "Access network discovery and selection in the evolved 3GPP multi-access system architecture," European Transactions on Telecommunications, vol. 21, no. 6, pp. 544-557, April 2010.

[14] S. Sharma, I. Baek, and T. Chiueh, "OmniCon: A mobile IP-based vertical handoff system for wireless LAN and GPRS links," Software: Practice and Experience, vol. 37, no. 7, pp. 779-798, 2007.

[15] C. Perkins, "IETF RFC 3344, IP Mobility Support for IPv4,” 2002.

[16] H. Soliman, "IETF RFC 5555, Mobile IPv6 Support for Dual Stack Hosts and Routers (DS-MIPv6)," June 2009.

[17] R. Wakikawa and S. Gundavelli, "IETF RFC 5844, IPv4 Support for Proxy Mobile IPv6 (DS-MIPv6)," May 2010.

[18] S. Gundavelli, K. Leun, V. Devarapalli, K. Chowdhury, and B. Patil, "IETF RFC 5213, Proxy Mobile IPv6 (PMIPv6)," August 2008.

[19] M. Bonola, S. Salsano, and A. Polidoro, "UPMT: universal per-application mobility management using tunnels," in Proc. IEEE Global Telecommun. Conf. (GLOBECOM), Honolulu, HI, USA, November 2009, pp. 2811-2818.

[20] J. Rosenberg, H. Schulzrinne, G. Camarillo, A. Johnston, J. Peterson, R. Sparks, M. Handley, and E. Schooler, "IETF RFC 3261, SIP: session initiation protocol," 2002.

[21] S. Salsano, A. Polidoro, C. Mingardi, S. Niccolini, and L. Veltri, "SIP-based mobility management in next generation networks," IEEE Wireless Communications, vol. 15, no. 2, pp. 92-99, April 2008.

[22] J.-Y. Song, S.-W. Lee, and D.-H. Cho, "Hybrid coupling scheme for UMTS and wireless LAN interworking," in Proc. IEEE Vehicular Tech. Conf. (VTC-Fall), vol. 4, Orlando, FL, USA, October 2003, pp. 2247-2251.

[23] M. Wasserman and P. Seite, "IETF Draft (Work in progress) - Current Practices for Multiple Interface Hosts," July 2011, Available at: http://tools.ietf.org/html/draft-ietf-mif-current-practices-12.

[24] S. Busanelli, M. Martalò, G. Ferrari, G. Spigoni, and N. Iotti, "Vertical handover between WiFi and UMTS networks: experimental performance analysis," International Journal of Energy, Information and Communications, vol. 2, no. 1, pp. 75-96, February 2011.

[25] M. Li, M. Claypool, and R. Kinicki, "Wbest: a bandwidth estimation tool for IEEE 802.11 wireless networks," in IEEE Conference on Local Computer Networks (LCN), Montreal, Canada, October 2008, pp. 374-381.

[26] Guglielmo S.r.l., Website: www.guglielmo.biz.

[27] B. Anton, B. Bullock, and J. Short, "Best current practices for wireless internet service provider (WISP) roaming,” Wi-Fi Alliance, Tech. Rep., February 2003.

[28] "Remote Access Service (RAS) Windows API," Available: http://msdn.microsoft.com/enus/library/bb545687(VS.85).aspx.

[29] R. Braden, “IETF RFC 1122, Requirements for Internet Hosts - Communication Layers,” 1989.

[30] Opnet Website, Website: http://www.opnet.com.

[31] D. B. Johnson and D. A. Maltz, "Dynamic source routing in ad hoc wireless networks," in Mobile Computing, T. Imielinski and H. Korth, Eds. Kluwer Academic Publishers, 1996, pp. 153-181.

[32] T. Jie, A. Klein, and D. Brown, "Natural cooperation in wireless networks," IEEE Signal Processing Mag., vol. 26, no. 5, pp. 98-106, September 2009.

[33] “Eureka project 6252 X-NETAD," Website: http://www.eurekanetwork.org/project/-/id/6252. 